\title{
Pedestrian Detection using Triple Laser Range Finders
}

\author{
Abdul Hadi Abd Rahman ${ }^{1}$, Khairul Akram Zainol Ariffin ${ }^{2}$, Nor Samsiah Sani ${ }^{3}$, and Hairi Zamzuri ${ }^{4}$ \\ ${ }^{1,2,3}$ Center for Artificial Intelligence Technology, Faculty of Information Science and Technology, , Universiti Kebangsaan \\ Malaysia. \\ ${ }^{4}$ Vehicle System Engineering Research Lab, Universiti Teknologi Malaysia, Jalan Semarak, 54100 Kuala Lumpur, Malaysia.
}

\begin{abstract}
Article Info
Article history:

Received: Jun 6, 2017

Revised: Aug 21, 2017

Accepted: Sep 3, 2017

Keyword:

Pedestrian Detection

Laser Range Finder

Autonomous

ABSTRACT

Pedestrian detection is one of the important features in autonomous ground vehicle (AGV). It ensures the capability for safety navigation in urban environment. Therefore, the detection accuracy became a crucial part which leads to implementation using Laser Range Finder (LRF) for better data representation. In this study, an improved laser configuration and fusion technique is introduced by implementation of triple LRFs in two layers with Pedestrian Data Analysis (PDA) to recognize multiple pedestrians. The PDA integrates various features from feature extraction process for all clusters and fusion of multiple layers for better recognition. The experiments were conducted in various occlusion scenarios such as intersection, closed-pedestrian and combine scenarios. The analysis of the laser fusion and PDA for all scenarios showed an improvement of detection where the pedestrians were represented by various detection categories which solve occlusion issues when low number of laser data were obtained.
\end{abstract}

Copyright (c) 2017 Institute of Advanced Engineering and Science. All rights reserved.

\section{Corresponding Author:}

Abdul Hadi Abd Rahman

Center for Artificial Intelligence Technology, Faculty of Information Science and Technology,

Universiti Kebangsaan Malaysia (UKM)

43600 UKM, Bangi Selangor, Malaysia

Phone: +603-8921 6712

Email: abdulhadi@ukm.edu.my

\section{INTRODUCTION}

Pedestrian Detection and Tracking (PDT) for autonomous ground vehicle has attracted more attention nowadays. A reliable PDT contributes to a significant improvement for other scenarios such as obstacle avoidance, path planning and collision avoidance. The presence of Laser Range Finder (LRF) which is capable of providing accurate range information, wide coverage area and a low time interval permits implementations in real time system. A reliable and efficient pedestrian detection in urban area is one of the crucial for successful autonomous navigation. Laser range finder provides valuable data of the surrounding especially for pedestrian detection but there are crucial limitations that need to be considered: a pedestrian could be segmented into several segments caused by partial occlusions and laser-absorbed such as glassy or black surfaces, and only parts of the objects facing the sensor are visible which often changes as the object moves which could degrade the detection result.

It was suggested that the LRF placement on a vehicle or robot is important in determining detection of body parts either waist or legs of pedestrians. Waist and legs are two of the most obvious features which could be very helpful in classification of a pedestrian especially in LRF implementation. Both implementation have their own advantages and drawbacks. A small leg size affects the amount of detected laser data especially in long range implementation thus may produce misclassification between leg and measurement noises. Meanwhile the waist part data may contain data of pedestrians hands which may sometimes cause occlusion for full waist data. The various orientation of pedestrian could easily have affected the detection misclassification and isolation of feature motion. A single planar approached using LRF is not sufficient enough for observing different object which are closed to each other $[1,2,3,4]$. The measurement quality of detected object is unequal. A high quality measurement is achieved when an object is in a clear view to the scanner. The measurement obtained is complete and good shape for further evaluation. Contradictly for block objects or when the sensor is blocked, it may be represented by partially 
and ambiguous shape [5,6]. The representation of pedestrian could be improved using multiple LRFs in multilayer implementation. It increased the possibilities for pedestrian detection in an environment for state estimation. However, it is challenging to handle data from various sources in term of data integration. There are factors that need to be considered such as sensor calibration, resource allocation and fusion technique while maintaining a low computation cost.

Thorpe [7] implemented a three LRFs configuration mounted on various positions placed at front and both sides of a vehicle to provide horizontal profiling while Wang [8] covered for both horizontal and vertical profiling. They suggested that pedestrian detection using laser scanners were difficult because the number of measurement points associated with a pedestrian is often small in the applications. Recognition algorithms can be used to confirm the results of lidar-based detection. Hashimoto et al. [9] further enhanced the configuration by allocating three LRFs in different layer to cover knee, thigh and waist part of pedestrian. A decentralised architecture approaches is chosen to provide a degree of scalability and robustness compared to centralised architecture. Sato et al. [10] has developed a pedestrian tracking method in various urban environments to improve the pedestrian detection rate for false/miss alarm using a six-layer-LRFs. Carballo [2, 11] implemented a fusion of two LRFs which arranged facing in opposite directions to cover $360^{\circ}$ surrounding. Then it was extended in multiple layers to create 3D model of people. Elliptical shape computed using Romanujanss approximation for chest area while small circular shapes detected in leg part. The centroid estimation extracted from the waist part was projected to lower body part to find correspond legs using average walking steps. The inclusion of reflection intensity data of LRF arranged in multiple layers was introduced in [12] for people detection. They included a calibrated intensity feature to the existing Adaboost to train better and strong classifiers. Carballo [13] further extended the fusion method by combining two LRFs in two layers to cover measurement of two different body parts. However, a reduction of 50 scan points has to be done for gathering simultaneous range and intensity from the multi LRFS. McKinley et al. [14] and Kim et al. [15] highlighted the use of multiple LRFs to improve the performance of the detection algorithms due to the increased amount of data for better robustness against occlusion. However, the scheme was highly dependent on the correct alignment of multi LRFs and could cause system failure if the misalignment was large enough. Mozos et al. [16] allocated three LRFs in different layers for detecting head, waist and leg parts of pedestrian. They assorted detection result by the level of confidence of each detected segment. A higher level confidence is defined when all pedestrian part is detected while low confidence referred to enough detection of any body parts. The approach was able to produce significant improvement to existing configurations. Gidel et al. [17] presented a pedestrian detection system using 4 horizontal plane layer of LRFs for false detections was reduced in comparison with application using a single laser scanner. An extremum map is computed by calculating all related probabilities of a pedestrian weighted by the intersections of number of layers. The fusion of the four layers of LRFs were executed in decentralised architecture. Experimental results proved that the usage of four laser planes has improved the pedestrian detection with a lower false alarms.

Improvement of detection approach is still an ongoing process. There are still limitations on the previous implementation for pedestrian detection. Previous researches as mentioned earlier showed that LRF configurations and placement is one of the factor that affected the detection performance. More specific application could be further explored for performance evaluation using LRF. There were less investigations on implementing LRFs in a multilayer configuration in outdoor environment to deal with high measurement noises. Therefore, this paper analyses on the performance enhancement in detection by proposing new laser fusion approach using multi LRFs in a multilayer configuration.

\section{RESEARCH METHODOLOGY}

In this study, Hokuyo Laser Range Finder (LRF) URG04-LX model has been selected as main sensor to provide environment data. The LRF was selected based on light-weight and easy to mount on any vehicle. It has a wide coverage area for LRF which are $240^{\circ}$ with $0.33^{\circ}$ angle resolution, maximum distance coverage of 5.6 meters in $0.1 \mathrm{sec}$ time interval. For that, a custom mounted for has been developed to place all LRFs as shown in Figure 1. The three LRFs were configured in two multilayer coverage. One LRF was placed at the center in front of the vehicle with height 1.2 meter to cover the width part of pedestrians. For bottom layer, two LRFs were positioned on both side in front of the vehicle at 0.4 meter above ground to produce an interlace of pedestrians legs data. The distance between right and left LRFs was set as 1.0 meter considering the vehicle width and mounting limitation.

The LRFs were filtered to cover $180^{\circ}$ angle and 5 meters in distance for all LRFS to cover focused area of targeted pedestrian. A set of calibration tests were done to ensure the accuracy of the produced data to represent legs $[18,19]$. The calibration results could not perform a $100 \%$ accuracy due to sensor noise of LRFs but it achieved considerably reliable output. The fusion technique to solve pedestrian detection in an outdoor environment from a ground moving vehicle was equipped with three Hokuyo Laser Range Finders (LRFs) which were configured in two different layers. The detection process involved a consecutive processing steps containing pre-process, pedestrian

IJECE Vol. 7, No. 6, December 2017: 3037 - 3045 


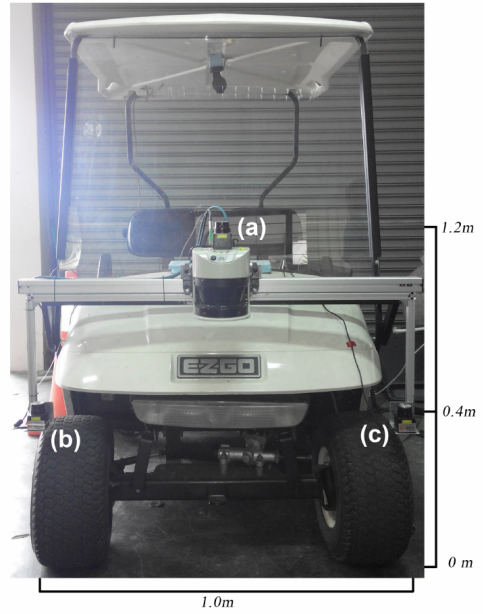

Figure 1. Mounted LRFs on vehicle platform where (a) LRF position on top center (b) LRF position on bottom right (c) LRF position on bottom left.

analysis, map matching and feature extraction. Raw data for each laser was pre-processed before being fused together. Then, the pedestrian data analysis (PDA) was performed to produce the observation output after passing the matching process with the developed online feature extraction mapping. There are four steps in pre-processing technique which includes data clustering, pedestrian analysis, map matching and feature extraction. The pre-processed (clustering) of streaming data for each LRF was executed using parallel processing technique. A two level fusion was proposed involving fusion of both LRFS at the bottom to calculate the distance of each extracted points for pedestrian legs. The details on fusion processing step are explained in Algorithm 1.

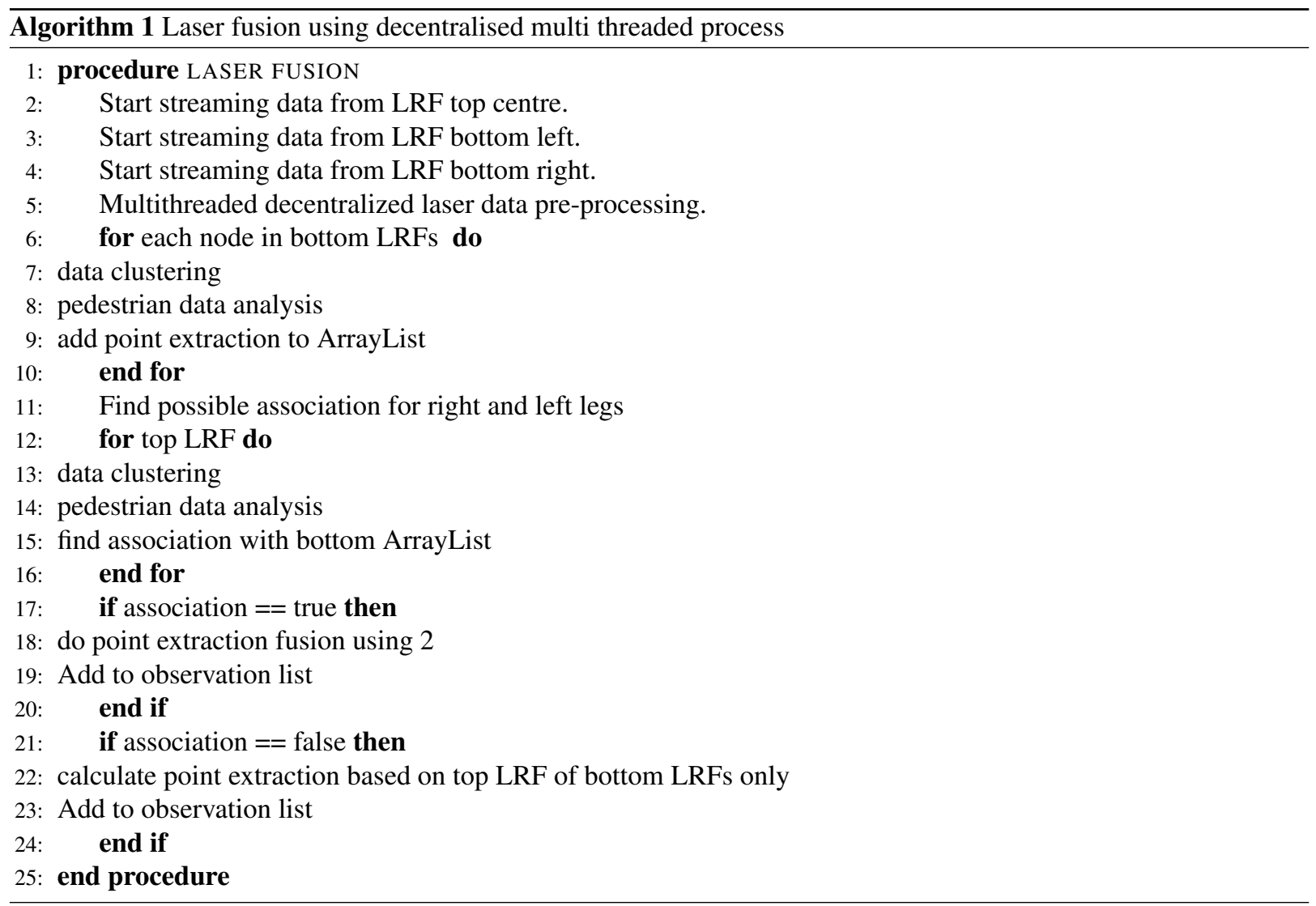


All clusters resulted from the previous process could possibly containing pedestrian or similar-like object. Further analysis for each cluster is required to determine the final observation for tracking process. This process is important to produce high quality observation while reducing the false alarm containing undesirable objects. There are few criteria have been identified to determine detection of pedestrian including feature analysis, integration of results for top and bottom laser, and occupancy grid in determine static or dynamic pedestrian. Based on conclusion and suggestion in Arras [5], few best features had been selected and evaluated for implementation for feature-based analysis for pedestrian detection described as follows; i) number of elements $(\mathrm{N})$ and width (W), curvature, mean angular difference, radius, boundary length, and multi-layer association. The Pedestrian Data Analysis (PDA) process is shown in Figure 2.

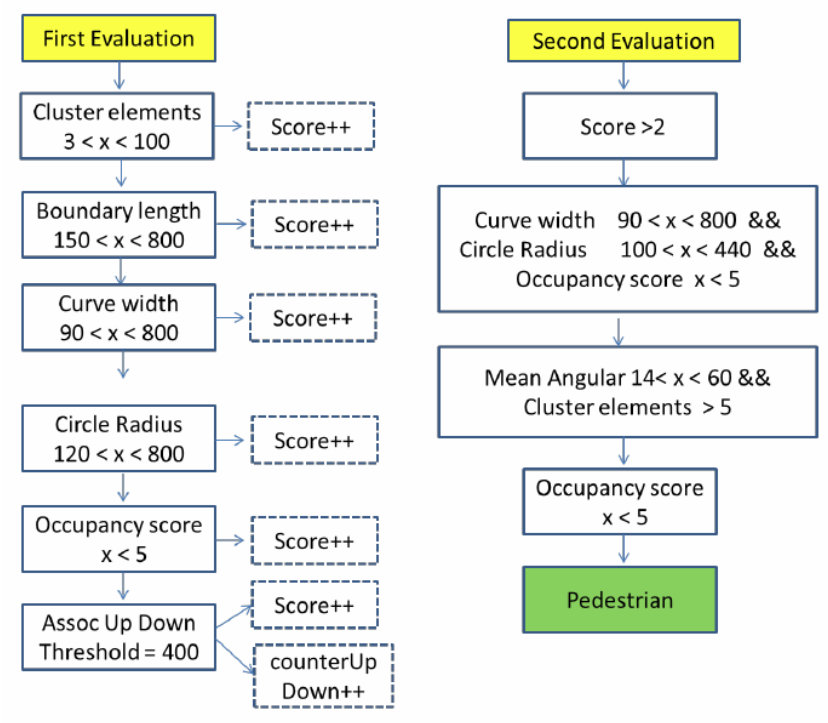

Figure 2. Pedestrian Data Analysis for pedestrian detection confirmation

\section{EVALUATION OF DETECTION AND FALSE RATE}

There are two most important parameters for pedestrian detection evaluation which are detection rate (DR) and false alarm rate (FAR). Detection rate represents the detection accuracy of the implemented approach. Implementation using 2 LRFs was chosen as benchmark for evaluation of the proposed approach. Since there are no ground truth data for the testing dataset, the analyses of the detection and false alarm rate were done manually for each testing scenario. In each frame of data, all moving objects for each data frame were identified with validation from the recorded video sequences. For each moving object, true and false detection were identified and counted for analysis. The parameters for Pedestrian Data Analysis (PDA) are described in previous section. This evaluation is important to get the best range for all parameters involved in determination of detected pedestrians based on the laser data input. A total number of 948 scans in an outdoor environment were collected where it involved pedestrian in both moving and standing still. The total number of segments extracted were 5349 segments. For each scan, the laser data was clustered and analyzed using the proposed pedestrian data analysis module. Results obtained from experiments using a modified Pedestrian Data Analysis and compared with commonly used approach from literature. Collection of data obtained from the experiment in both static and moving vehicle using the proposed configuration of 3 LRF were compared with previous selected multilayer implementation. Table 1 lists the results of the analysis has been sorted based on PDA into 5 categories as follows: C1 - waist with one leg for either one of the bottom LRFs, C2 - waist with one leg for both LRFs bottom, C3 - waist with two legs for either one of the bottom LRF, C4 - waist with two legs for both bottom LRFs and C5 - waist only. From Pedestrian Data Analysis (PDA) results, it can be concluded that the most informative feature is the radius of the circle that fitted into the segment. The mean angular difference is the second most important feature which quantified the convexity of the segment. The combination of curvature and radius does not measure the degree of circularity but provide extra information of detected pedestrians.

IJECE Vol. 7, No. 6, December 2017: 3037 - 3045 
Table 1. Average values for all parameters in pedestrian data analysis.

\begin{tabular}{ccccccc}
\hline & $\begin{array}{c}\text { total } \\
\text { clusters }\end{array}$ & $\begin{array}{c}\text { cluster } \\
\text { element }\end{array}$ & $\begin{array}{c}\text { cluster } \\
\text { width }(\mathrm{mm})\end{array}$ & $\begin{array}{c}\text { boundary } \\
\text { length }(\mathrm{mm})\end{array}$ & $\begin{array}{c}\text { mean } \\
\text { angular }\left({ }^{\circ}\right)\end{array}$ & $\begin{array}{c}\text { circle } \\
\text { radius }(\mathrm{mm})\end{array}$ \\
\hline C1 & 711 & 20 & 495 & 617 & 54.9 & 183 \\
$\mathrm{C} 2$ & 1095 & 17 & 532 & 636 & 62.3 & 188 \\
$\mathrm{C} 3$ & 828 & 24 & 619 & 613 & 42 & 182 \\
$\mathrm{C} 4$ & 1842 & 22 & 600 & 669 & 53 & 183 \\
$\mathrm{C} 5$ & 873 & 13 & 523 & 513 & 59.4 & 186 \\
\hline
\end{tabular}

\subsection{Pedestrian Detection Evaluation}

The pedestrian detection and tracking during intersection was performed to demonstrate the pedestrian identification and track association capabilities. Two method were involved in this evaluation. Conventional laser configuration (2LRF) represents combination of laser configuration using 2 LRFs and existing conventional Multiple Hypothesis Tracking (MHT) approach. The proposed laser configurations (3LRF) approach consists of laser fusion using 3 LRFs in two layers with an improve MHT method from previous research in [19]. In the simulation and experiment, a multiple pedestrian situation from different direction and velocity with intersecting trajectory is simulated and experimented. To ensure the effectiveness of the propose dyMHT algorithm, the evaluation on the performance were conducted separately before any further tests were proceed. The proposed tracking algorithm were evaluated in two experiments. First, the experiments were conducted for separate conditions for intersection and closed-pedestrian. For intersection scenarios, a series of repeated experiments were arranged to produce pedestrian intersections. To encourage frequent track crossings, the pathway for targets were defined which involve pedestrians velocity state components which slightly bias toward the each of others. Each scenario was simulated and experimented in three repeated tests. It was expected that the algorithm was able to track all pedestrian in all intersection scenarios. Then, the experiment for detection for closed-pedestrians (multi-pedestrian walking side by side) were conducted in which more complicated compared to intersection cases due to uncertain occlusion interval depending on pedestrian walking pattern and direction.

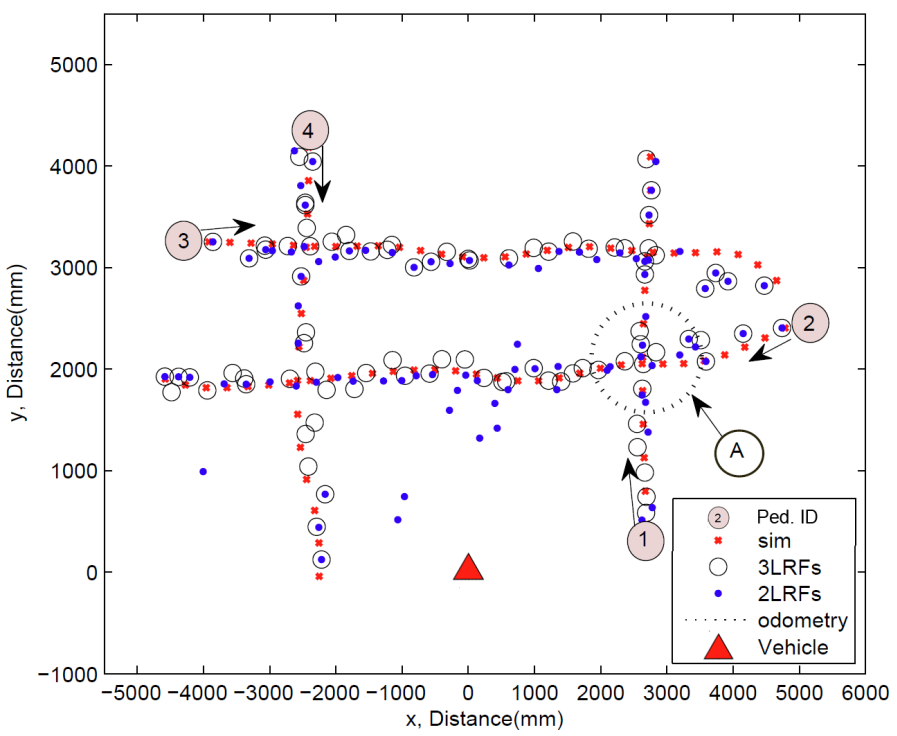

Figure 3. Scenario 1: Pedestrian Detection during Intersection Cases.

Figure 3 shows the detection results for moving pedestrian during intersection. The simulation data for this scenario is represented by ' $x$ ' marker for benchmarking purposes. It is observed that at certain parts of the detection, the implementation using 3 LFRs was able to produce more observations result based the plotted point for Point Extraction (PE) compared to 2 LRFs approach. It is supported by the average detection rate which obtained during intersection scenarios using the proposed approach which was $98.9 \%$ compared to $92.6 \%$ using the benchmark approach. Total of $4.3 \%$ reduction of false alarm was achieved for the proposed approach. In general, improved detection results were observed compared to benchmark approach which produced lower false alarm due to imple- 
mented occupancy grid and multi clustering during fusion process. The intersection detection result which described the situation is highlighted in circle labelled A in Figure 3.

It shows two pedestrians on the right walked across each other which caused intersection to happen. Before intersection happened, both approaches were able to detect both pedestrian. The front pedestrian was well spotted with different point extraction while the second pedestrian only detected by top laser. During intersection, the proposed approach was able to detect the occluded pedestrian as opposed to benchmark method. It remained for a few iterations before the benchmark approach able to redetect the occluded objects. The recorded pedestrian data for $2 \mathrm{LRF}$ approach was less accurate to be classify as pedestrian during PDA process, since the occluded pedestrian was only detected after a few iterations when partial occlusion decreased. Meanwhile, the proposed approach was successfully deal with this problem where the generated pedestrian data obtained with the additional LRF allowed a better detection results.

The second experiment evaluated the capability of the proposed method to deal with group pedestrians walking closed to each other which produced a complex occlusion scenario. Figure 4 shows the detection results of five consistent pedestrians for closed scenario where pedestrian moving closed to each other which led to occlusion. It is observed that in 2LRFs approach, Pedestrians with ID \#4 and \#5 suffered from occlusion at the end of the pathway. The proposed configuration was able to recover more detections for Pedestrians \#5. The detection and false alarm rate for this scenario obtained a higher average detection rate at $89.6 \%$ was achieved in closed scenarios using the proposed approach compared to 78.7\% using the benchmark approach, 2LRFs. Both approaches (3 LRFs and 2 LRFs) produced the same false alarm rate at $4.2 \%$. The pre-post detection results for closed-pedestrian scenario were as labelled B in Figure 4. It shows two pedestrians on the right walked across each other which caused occlusion to happen. Before occlusion happened, both approaches were able to detect all pedestrians. The front pedestrian was spotted with different point extraction while the second pedestrian only detected by top laser. During, the proposed approach was not able to detect the occluded pedestrian similar to benchmark method. It lasted for a few iterations before the benchmark approach able to redetect the two occluded objects but never recovered the third occluded pedestrian.

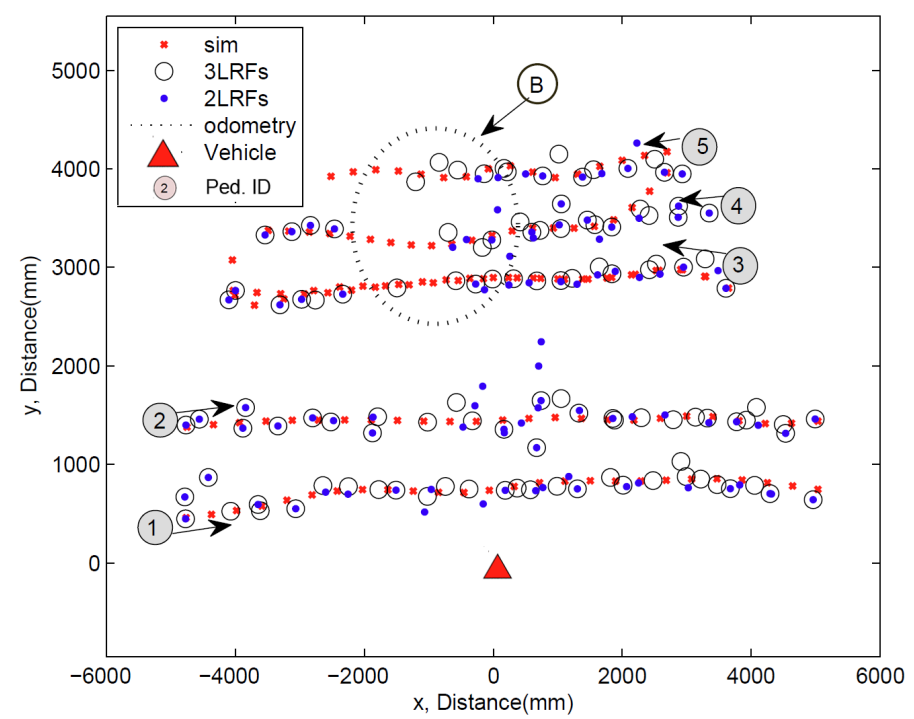

Figure 4. Scenario 2: Pedestrian Detection during Closed-pedestrian Cases

The detection results using fusion of LRFs for combine case scenarios are shown in Figure 5 where two closed pedestrian scenarios ( 2 pedestrians each) and 2 intersection cases. The detection of five pedestrians were found correctly with some occlusions remain appeared in tracking process. The average detection rate at $93.5 \%$ was achieved in this scenario using the proposed approach compared to $78.2 \%$ using the benchmark approach. The false alarm achieved for both approaches (3 LRFs and $2 \mathrm{LRFs}$ ) were at 5.1\%.

\subsection{Effect of Laser Fusion in Pedestrian Detection}

The approach presented in this research described a multi-part person detection based on multiple 2D LRF scans. The first highlight in pedestrians detection using laser scanners is the position of the lasers. For the experiments presented in this research, the lasers were placed at two different fixed heights. These heights were selected to cover 


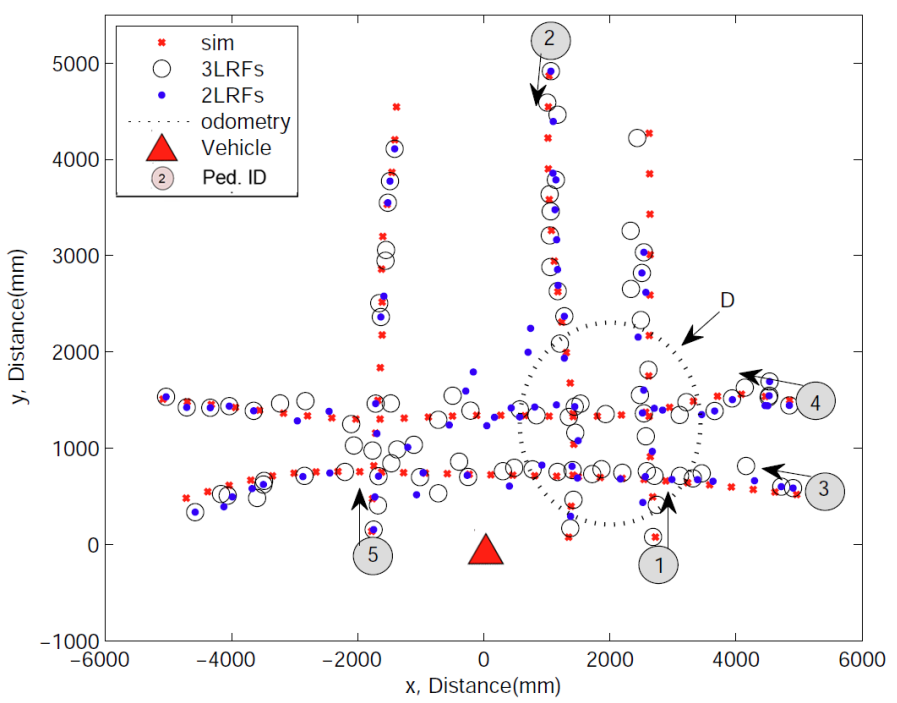

Figure 5. Scenario 3: Pedestrian detection during Combine Intersection and Closed-pedestrian

a range of $400 \mathrm{~mm}$ for feet and $1200 \mathrm{~mm}$ for waist. However, in this research, it has been find out that 2 layers with extra LRF unit for bottom layer to cover feet level were enough to improvise the pedestrian detection. The detection results produced in all four experiments proved that better detections accuracies were achieved. This outcome has been supported by a research conducted by Carballo et al. [14] and Mozos et al. [17] who have performed a fusion of multiple layers by segmentation of fused scan data, geometrical features extraction and association for every detected person to allow good position estimation and prediction pedestrians direction. The combination of both areas creates a 3D volume which helps locating the position of the person more closely related to the center of detected pedestrian.

The experimental results found that the proposed LRF configurations were able to increase the detection rate and lower or same false alarm rate in all given scenarios. The Pedestrian Data Analysis (PDA) was applied to solve the misclassification rates thus achieving lower false alarm rate. In comparison, Carballo et al. [13] found that laser intensity was able to improve the detection results in the single-layer system. However only minimal false alarm rate reduction was achieved, but highlighting about detection rates with smaller misclassification rates. Another issue highlighted by was the drawback to get simultaneously range and intensity from multi-sensors, where higher angular resolution was used, contributing to $50 \%$ reduction of total scan points. To solve the problem, an optimised parallel processing was implemented for fusion of all laser data with full angle resolution. The experiments were conducted to solve several critical scenarios. It involved people walk across, the trajectory of each person intersect each other which causing detections fragmented into several parts. In the scenario where laser tracking fails due to the data confusion when people walk close together, that situation is very difficult to deal with. The situation is complex where the laser data of the bottom person is lost due to occlusion by other people. The tracking process depended on the direction and speed of upper person which treated as group tracking due to the high confidence in similarity of movement and pattern. Furthermore, combine case scenarios were conducted for performance evaluation. The presented fusion of LRFs worked fairly well with much better performance when the vehicle was static than when the vehicle moved as seen in the experimental results. The use of multiple LRFs improved the performance of the detection algorithms due to the increased amount of data and made object tracking more robust against occlusion. However, the scheme was highly dependent on the correct alignment of the two bottom LRFs and could cause system failure if the misalignment was large enough. For that, in this research, a series of verification and calibration of the fusion result were done before running the experiments. The average detection rate for all experiments for the proposed fusion method was recorded at $92.5 \%$ which is an increment of $9.9 \%$ from the benchmark approach. The average false alarm rate for both implementations were $5 \%$ and $5.9 \%$ which represents $(0.9 \%)$ of reduction.

\section{CONCLUSION}

This paper presented the experimental results, analysis and discussion for the proposed configuration for fusion of three LRFs using pedestrian data analysis. It is shown that it was able to achieve better detection results and assure detection of static objects. The experimental results in different outdoor scenarios showed an increment in pedestrian detection accuracy compared to implementation using double layer of two LRFs. 


\section{ACKNOWLEDGEMENT}

The authors would like to thank the Malaysia-Japan International Institute of Technology (MJIIT) in Universiti Teknologi Malaysia for the support and funding of this research.

\section{REFERENCES}

[1] X. Shao, H. Zhao, K. Nakamura, K. Katabira, R. Shibasaki, and Y. Nakagawa, "Detection and Tracking of Multiple Pedestrians by Using Laser Range Scanners," in IEEE/RSJ International Conference on Intelligent Robots and Systems, no. 1. IEEE, 2007, pp. 2174-2179.

[2] A. Carballo, A. Ohya, and S. Yuta, "Fusion of double layered multiple laser range finders for people detection from a mobile robot." IEEE, Aug. 2008, pp. 677-682. [Online]. Available: http://ieeexplore.ieee.org/lpdocs/epic03/wrapper.htm?arnumber=4648023

[3] Y. Yakiyama, N. Thepvilojanapong, M. Iwai, O. Mihirogi, K. Umeda, and Y. Tobe, AIPSJ Online Transactions, vol. 4, pp. 515-528, 2009.

[4] L. Adiaviakoye, P. Patrick, B. Marc, and J. M. Auberlet, "Tracking of multiple people in crowds using laser range scanners," in 2014 IEEE Ninth International Conference on Intelligent Sensors, Sensor Networks and Information Processing (ISSNIP), April 2014, pp. 1-6.

[5] K. O. Arras, O. M. Mozos, and W. Burgard, "Using Boosted Features for the Detection of People in 2D Range Data," in Proceedings 2007 IEEE International Conference on Robotics and Automation. IEEE, Apr. 2007, pp. 3402-3407. [Online]. Available: http://ieeexplore.ieee.org/lpdocs/epic03/wrapper.htm?arnumber=4209616

[6] T. Zhao, R. Nevatia, and B. Wu, Segmentation and tracking of multiple humans in crowded environments, IEEE Transactions on Pattern Analysis and Machine Intelligence, vol. 30, pp. 1198-211, 2008.

[7] C. Thorpe and A. Suppe, "LADAR-based detection and tracking of moving objects from a ground vehicle at high speeds," in IEEE IV2003 Intelligent Vehicles Symposium. Proceedings (Cat. No.03TH8683). IEEE, 2003, pp. 416-421. [Online]. Available: http://ieeexplore.ieee.org/lpdocs/epic03/wrapper.htm?arnumber=4209616

[8] C.-C. Wang, "Simultaneous Localization, Mapping And Moving Object Tracking,” Ph.D. dissertation, Carnegie Mellon University, 2004.

[9] M. Hashimoto, Y. Matsui, and K. Takahashi, "Moving-object tracking with multi-laser range sensors for mobile robot navigation," in 2007 IEEE International Conference on Robotics and Biomimetics (ROBIO). IEEE, 2007, pp. 399-404.

[10] S. Sato, M. Hashimoto, M. Takita, K. Takagi, and T. Ogawa, "Multilayer lidar-based pedestrian tracking in urban environments," in 2010 IEEE Intelligent Vehicles Symposium. IEEE, Jun. 2010, pp. 849-854. [Online]. Available: http://ieeexplore.ieee.org/lpdocs/epic03/wrapper.htm?arnumber=5548135

[11] A. Carballo, A. Ohya, and S. Yuta, "People detection using range and intensity data from multi-layered Laser Range Finders," in 2010 IEEE/RSJ International Conference on Intelligent Robots and Systems. IEEE, Oct. 2010, pp. 5849-5854. [Online]. Available: http://ieeexplore.ieee.org/lpdocs/epic03/wrapper.htm?arnumber=5649769

[12] — - "Laser reflection intensity and multi-layered Laser Range Finders for people detection," in 19th International Symposium in Robot and Human Interactive Communication. IEEE, 2010, pp. 379-384. [Online]. Available: http://ieeexplore.ieee.org/lpdocs/epic03/wrapper.htm?arnumber=5649769

[13] _ - Reliable People Detection Using Range and Intensity Data from Multiple Layers of Laser Range Finders on a Mobile Robot, International Journal of Social Robotics, vol. 3, pp. 167-186, 2011.

[14] N. McKinley, "Simultaneous Localization, Mappings and Object Tracking in an Urban Environment using Multiple 2D Laser Scanners,” Ph.D. dissertation, 2010.

[15] B. Kim, B. Choi, M. Yoo, H. Kim, and E. Kim, Robust Object Segmentation Using a Multi-Layer Laser Scanner, Sensors, vol. 14(11), pp. 20400-20418, 2014. 
[16] O. M. Mozos, R. Kurazume, and T. Hasegawa, Multi-Part People Detection Using 2D Range Data, International Journal of Social Robotics, vol. 2, pp. 31-40, 2010.

[17] S. Gidel, P. Checchin, C. Blanc, T. Chateau, and L. Trassoudaine, Pedestrian Detection and Tracking in an Urban Environment Using a Multilayer Laser Scanner, vol. 11, pp. 579-588, 2010.

[18] A. H. A. Rahman, H. Zamzuri, S. A. Mazlan, and M. A. A. Rahman, "Model-Based Detection and Tracking of Single Moving Object Using Laser Range Finder," in 2014 5th International Conference on Intelligent Systems, Modelling and Simulation. IEEE, jan 2014, pp. 556-561. [Online]. Available: http://ieeexplore.ieee.org/lpdocs/epic03/wrapper.htm?arnumber=7280971

[19] A. Rahman, H. Zamzuri, and S. A. Mazlan, Dynamic Track Management in MHT for Pedestrian Tracking Using Laser Range Finder, Mathematical Problems in Engineering, vol. 2015, pp. 1-9, 2015.

[20] S. Gidel, P. Checchin, C. Blanc, T. Chateau, L. Trassoudaine, and U. B. Pascal, "Pedestrian Detection Method using a Multilayer Laserscanner : Application in Urban Environment," in 2008 IEEE/RSJ International Conference on Intelligent Robots and Systems. IEEE, 2008, pp. 22-26.

\section{BIOGRAPHIES OF AUTHORS}
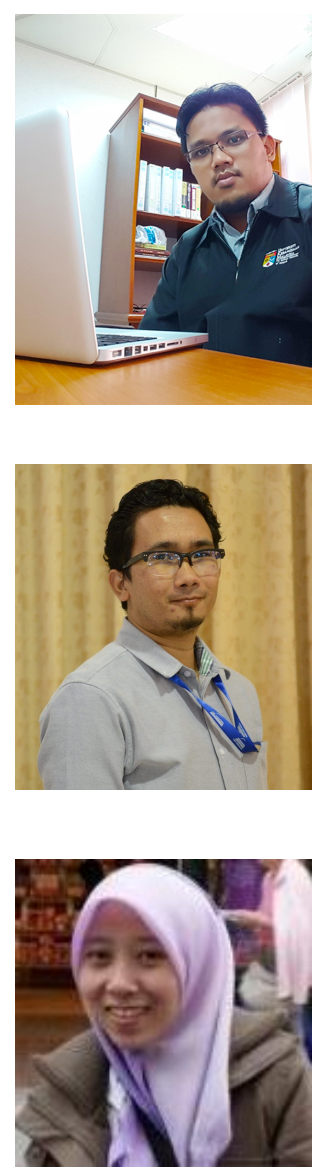

Abdul Hadi Abd Rahman is a senior lecturer at Center for Artificial Intelligence Technology, Faculty of Information Science and Technology, Universiti Kebangsaan Malaysia. He obtained Doctor of Philosophy from Universiti Teknologi Malaysia. His researches are in fields of object tracking and artificial intelligence. Further info on his homepage: http://www.ftsm.ukm.my/hadi

Khairul Akram Zainol Ariffin is a senior lecturer at Center for Software Technology and Management, Faculty of Information Science and Technology, Universiti Kebangsaan Malaysia. He obtained Doctor of Philosophy from Universiti Teknologi Petronas. His researches are in fields of networking and cyber security.

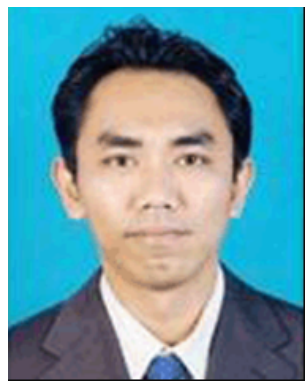

Hairi Zamzuri is an Associate Professor at Malaysia-Japan International Institute of Technology (MJIIT), Universiti Teknologi Malaysia. His researches are in fields of vehicle dynamic, railway

vehicle, autonomous vehicle, vehicle suspension design.

Nor Samsiah Sani is a senior lecturer at Center for Artificial Intelligence Technology, Faculty of Information Science and Technology, Universiti Kebangsaan Malaysia. His researches are in fields of machine learning and artificial intelligence. 